\title{
Implementation Challenges of Self Service Business Intelligence: A Literature Review
}

\author{
Christian Lennerholt \\ University of Skövde \\ christian.lennerholt@his.se
}

\author{
Joeri van Laere \\ University of Skövde \\ joeri.van.laere@his.se
}

\author{
Eva Söderström \\ University of Skövde \\ eva.soderstrom@his.se
}

\begin{abstract}
In a traditional Business Intelligence (BI) system, power users serve less experienced casual users. Power users analyze and gather data requested by casual users, and produce the reports and visualizations that casual users base their decisions on. When data volumes and the usage frequency of $a$ traditional BI system increase, power users have problems serving all the requests from casual users. The Self Service Business Intelligence (SSBI) approach can enable users to be more self-reliant and less dependent on power users. Although SSBI promises more benefits compared to a traditional BI system, many organizations fail to implement SSBI. The literature review presented in this paper discusses six SSBI challenges related to "Access and use of data" and four challenges related to "Self-reliant users". Awareness of these ten challenges can help practitioners avoid common pitfalls, when implementing SSBI, as well as guide SSBI researchers in focusing on their future research efforts.
\end{abstract}

\section{Introduction}

Today, Business Intelligence (BI) is widely used by many organizations, since it helps them make better decisions on time [1, 2]. The BI collection of technologies and software enables organizations to be innovative, creative and competitive. In order to make better decisions, users need a solid foundation of data that is available and accessible at all times within the entire organization, from strategic to operational levels. In a traditional BI system, there is a request and response relationship between users. Decision makers issue a request to technical power users (BI experts) to create reports that visualize data needed by decision makers in order to make good decisions on time [3].

According to Logi Analytics [1] and their 2015 state of SSBI report, which is based on an online survey of more than 800 business and technology professionals, the traditional BI approaches are changing. Instead of focusing on supporting power users, organizations in general must be able to work with data more easily. According to $91 \%$ of the survey's respondents, easy access to data, without help from IT departments and power users, is needed. In response to this demand, the new and promising BI trend with long-term prospects is Self-Service Business Intelligence (SSBI) [4]. According to Logi Analytics [1], 95\% of the survey's respondents plan to invest in SSBI within the next two years.

The SSBI approach enables users to be more selfreliant. It allows all users to access data and to conduct their own analyses for decision-making, without the need to engage an IT department and power users. Reports that could take months to deliver can now be produced on the fly [2]. The most compelling motivation for implementing SSBI is the increased flexibility it offers users, making them more selfreliant and thus improving the operational efficiency of organizations.

Nonetheless, the implementation rate of SSBI is low, even though its advocates claim that it is the future of $\mathrm{BI}$ and promises more advantages compared to the traditional approach. According to a survey of 234 respondents, conducted by Eckerson [5], two thirds, (64\%), of the $70 \%$ that are qualified BI professionals, rate their success with the SSBI approach as average or lower, while more than half (52\%) of the newcomers rate their attempts at SSBI as fair or lower. According to Logi Analytics [1], only $22 \%$ of the respondents have access to data when needed [1]. Such results highlight the fact that in practice, implementing SSBI is not as easy as expected $[1,3,4,5,6,7]$. Several authors $[3,8]$ argue that more detailed research on how organizations can implement SSBI is needed. This paper aims to identify challenges associated with the implementation of SSBI, thereby creating an awareness of such challenges which will enable organizations to better prepare for their transition phase and avoid potential pitfalls. 


\section{Background}

The heart of BI is often considered a data warehouse that stores all relevant internal and external data [9]. The sources of data vary in quality and format, and have different meanings, depending on their origin. Today, data has a finer granularity and is generated in much larger volumes than before. ETLtools (Extract, Transform and Load) are normally employed to integrate this data and to make sure it is valid and useful for an organization. In addition, ETLtools clean the data for storage in the data warehouse and prepare it for BI tasks. These processes refer to the back-end of a traditional BI system [9]. When data is available in the central data warehouse, different midtier servers are used for data analysis, which is the foundation of the decision reports used by decision makers. This process refers to the front-end of a traditional BI system [9].

In order to make the entire BI system operational, there are different kinds of power and casual users [3, 5]. Power users are the BI experts that retrieve the data required to generate the reports that are used as a foundation for decision-making. Managers and decision makers are normally the casual users that make decisions within an organization on the basis of these reports. Casual users normally have less experience of data usage and analysis, compared to power users.

When casual users of a traditional BI system need to make decisions, they ask power users to create a report that visualizes and simplifies the process of making decisions. Power users interpret the requests from casual users, combine the needed data, perform a customized analysis and then produce a report that visualizes the result. If the casual users want to view the report from a different angle, this request/response process is repeated, making the entire procedure a time-consuming undertaking.

Since ever more data from internal and external sources is being stored in the data warehouse, and more users are applying the BI system, the request/response process between power and casual users is exerting much more stress on the IT department to deliver reports on time [3, 4]. The resulting bottleneck in the communication between power and casual users can therefore hinder time critical decisions. As a result, casual users may make time critical decisions without processing all the available data in the reports. According to Imhoff and White [2], many casual users in organizations make decisions that are not based on $\mathrm{BI}$, due to the power users' inability to deliver reports on time. Furthermore, due to cost-saving measures, many IT departments have been reduced, even though the number of casual users is increasing and the need for more access to BI and its available data is growing among the increasing number of casuals users [2].

The SSBI approach is the result of the increase in available data and users of a BI system, and is considered the current trend in BI development [4]. The aim of SSBI is to establish a BI system that enables casual users to make decisions without help from power users. Casual users should be able to access and query data, use predefined reports, analyze data or create their own reports, in order to make decisions on time. The SSBI process enables casual users to be more self-reliant and less dependent on the power user. Imhoff and White [2, page 4] define SSBI as: "The facilities within the BI environment that enable BI users to become more self-reliant and less dependent on the IT organization. These facilities focus on four main objectives: easier access to source data for reporting and analysis, easier and improved support for data analysis features, faster deployment options such as appliances and cloud computing, and simpler, customizable, and collaborative end-user interfaces." This definition of SSBI is used in this paper, since it focuses on simplifying the process of BI use.

\section{Research method and analysis}

A wide range of strategies can be used to conduct research in information systems [10]. One such strategy, which is also regarded as an important and valuable research method [11,12, 13], is a systematic literature review in the field of information systems. Many researchers argue that most studies in the field of information systems are predominantly empirical and that more literature studies are needed $[11,12,13]$. A literature review helps to integrate the contributions from empirical research studies into a coherent whole and can accelerate theoretical progress in a research field [11, 12, 14].

Webster and Watson [12] have published a wellknown paper that presents a discussion on how to conduct a literature review in the field of information systems. To achieve even more precision and rigor in the literature analysis process, Wolfswinkel et al. [14] extend the guidelines of Webster and Watson by combining them with Grounded Theory. The approach achieved as a result is a high-quality tool which allows concepts to emerge from the literature in an inductive way [14]. The aim of this paper is to conduct a systematic literature review using Grounded Theory as the analysis method, as in [14]. 


\subsection{Literature search}

The literature review was conducted in the spring of 2017 and included literature that relates to the field of SSBI. Various search engines were used to find the literature, such as WorldCat, Google Scholar, Semantic Scholar and Web of Science. These search engines have access to multiple databases, such as ACM Digital Library, IEEE Xplore, ScienceDirect, ArXiv, DBLP, CiteSeer, OdySci, Academic, AMiner, Scopus, EBSCOhost and Emeraldinsight.

The following search string, which targets the title, abstract and keywords, was used in the literature review: "Business Intelligence" OR BI AND "Self Service” OR SSBI. However, not all search engines and databases use the same logical syntax of OR and AND. Therefore, the search string was adapted to fit the syntax of each search engine and database.

Literature was selected on the basis of the fulfillment of the following criteria: a) written in the English language, b) published in a peer-reviewed scientific journal or conference publication, in state of the art or best practice reports, or in textbooks. The search process found 110 articles (duplicates included), however, a total of 21 articles remained after duplicates and those that did not accord with the selection criteria were removed. Forward and backward citations were checked and their lists of references were used to find additional relevant literature. However, it was concluded that enough literature had already been found in the initial search.

\subsection{Qualitative analysis}

A qualitative analysis approach that applied open, axial and selective coding was used iteratively to analyze the literature [14]. The goal was to inductively identify a set of main categories and sub-categories that demonstrate how the literature portrays SSBI implementation challenges [14].

The analysis process used to identify categories in the literature that are relevant to the research question is defined as theme analysis [10]. Different segments of text identified as a challenge were grouped into categories. Thereafter, the categories were named to appropriately reflect the identified groups of challenges. Categories identified in the literature data were developed using an inductive approach, which means that categories were created with an open mind and not according to any predefined theories of challenges [10, 14].

The analysis of the literature review was an iterative process in which categories were refined in several steps to capture all of the identified challenges.
More specifically, the analytical process of open coding involved reviewing the selected literature by going back and forth between the papers, in order to generate a high level of abstraction of categories [14]. The aim of the open coding was to iteratively identify a set of categories that illustrate how the literature relates to all identified challenges of SSBI implementation. Axial coding was used iteratively to find subcategories and properties that relate to the main category. Selective coding was used iteratively to go back and forth between the literature, in order to integrate and refine the identified categories. The creation of these categories was an iterative process. As more literature was analyzed, current categories were refined, either by merging, separating or relabeling them. In addition, already processed papers were re-analyzed to ascertain whether they could be included in the current categories. When all the literature had been analyzed and no new categories could be identified, the analysis of the literature was deemed saturated and considered completed [14].

The review of the 21 papers, conducted in order to identify challenges of SSBI implementation, found 14 articles that include contributions related to the research question. The remaining 7 articles include no such challenges and, thus, have not contributed to the results.

\section{SSBI implementation challenges}

The result of the literature analysis is summarized in table 1. It presents challenges of SSBI implementation that have been identified inductively, using open, axial and selective coding, and shows how the analyzed literature relates to the challenges.

Table 1: SSBI implementation challenges

\begin{tabular}{|c|c|}
\hline SSBI implementation challenges & References \\
\hline \multicolumn{2}{|l|}{ Access and use of data } \\
\hline \#1.1: Make data sources easy to access and use & $\begin{array}{l}{[1,2,6,7,15,} \\
16,17,18,19]\end{array}$ \\
\hline \#1.2: Identify data selection criteria & {$[7,15,16,20]$} \\
\hline \#1.3: Use correct data queries & {$[7,15,16,20]$} \\
\hline $\begin{array}{l}\text { \#1.4: Control of data integrity, security and } \\
\text { distribution }\end{array}$ & {$[1,3]$} \\
\hline $\begin{array}{l}\text { \#1.5: Define policies for data management and } \\
\text { data governance }\end{array}$ & {$[6,7,16]$} \\
\hline \#1.6: Prepare data for visual analytics & {$[6,8]$} \\
\hline \multicolumn{2}{|l|}{ Self-reliant users } \\
\hline \#2.1: Make BI tools easy to use & {$[2,3,6,15,19]$} \\
\hline $\begin{array}{l}\text { \#2.2: Make BI results easy to consume and } \\
\text { enhance }\end{array}$ & {$[2,3,6,15]$} \\
\hline \#2.3: Give the right tools to the right user & {$[3,5,6,21]$} \\
\hline $\begin{array}{l}\text { \#2.4: Educate users in how to select, interpret } \\
\text { and analyze data for decision- making }\end{array}$ & {$[5,6,8]$} \\
\hline
\end{tabular}


The rest of section 4 describes the identified categories: sub-section 4.1 presents the main categories, while 4.2 and 4.3 describe the subcategories.

\subsection{Main categories of SSBI implementation challenges}

In the coding analysis process, two main categories were identified: "Access and use of data" and "Selfreliant users". Challenges that are caused by issues related to data access and usage were grouped into six sub-categories, while challenges related to users becoming more self-reliant formed four sub-categories.

According to the definition of SSBI used in this paper, the purpose of SSBI is to simplify the process of BI use. Casual users will become self-reliant and will be able to access data without depending on the IT department, while power users will be able to accomplish their tasks more quickly and efficiently [3]. The main categories of challenges identified in the literature reflect the definition of SSBI, i.e., enable users to be more self-reliant and give them direct access to data as desired.

All users have different demands and skills when they apply BI tools. Those with the appropriate skills do not need to wait for power users to prepare their request for data. When implementing SSBI, a crucial challenge to overcome is allowing users to be more self-reliant by enabling them to access, use and consume data without asking power users for help [3]. A major driver for implementing SSBI is its ability to empower casual users to quickly and independently perform analytics without help from power users [2, 3]. According to Imhoff and White [2], 47\% of the 587 respondents in their study want to create their own reports without waiting for power users to respond to their request. For users to make better decisions, SSBI constitutes a significant number of methods and tools that can improve the decision-making process. However, it requires that users have easy access to data at the right time and in the right format, and that the data is easy to use and consume, compared to traditional BI methods. In order for users to be more self-reliant and not need the help of power users, the following challenges must be overcome: make the retrieval and use of existing data flexible, remove the difficulty of using multiple data sources, and enable multiple options for different data use and consumption [3]. SSBI should be an environment that facilitates the access, use and sharing of data, information, reports and analysis. The following sub-sections describe the identified challenges in the two main categories "Access and use of data" and "Self-reliant users".

\subsection{Access and use of data}

This section describes the six identified challenges in the main category: "Access and use of data".

\section{Challenge \#1.1: Make data sources easy to access and use}

The volumes of available data that are of interest to many organizations, normally referred to as "Big Data” , are increasing significantly [17]. Therefore, to be able to embrace this new available data, the traditional data warehouses, which are normally external, must be expanded. Users need the ability to use data freely, in order to gain new insights, analyze data and modify reports, as they desire [1, 7]. In a traditional BI system, power users deliver BI solutions to casual users, which is a time-consuming process [3]. One SSBI implementation challenge is that access to data sources must be simplified and accelerated. Furthermore, users should be able to integrate structured and unstructured data easily and in a selfservice manner, without help from power users $[1,3$, 6]. According to the TDWI best practice report by Stodder [6], most users lack the ability to use data freely and in a self-service manner. There must be an organizational process that facilitates the modification of standard reports [1]. The ability to quickly access, deploy and manage data warehouses increases the productivity of users. Instead of helping casual users, power users could then focus on delivering BI solutions that can be used in a self-service manner, which increases the value of BI [2, 3, 6, 17].

\section{Challenge \#1.2: Identify data selection criteria}

To perform a correct analysis, it is important to ensure the quality of the data used. Therefore, data should be selected according to specific criteria regarding quality. One of the challenges to overcome is determining the quality criteria that should be considered in the data selection process, especially regarding data from external sources. When retrieving external data, there is a tradeoff between quantity and quality.

Abelló, et al. [20] propose a number of criteria that could be used to address data quality:

- Relevance to keywords: Criteria regarding data retrieval should include metadata and tags that define the source data.

- Integrability: The quality of the external data is higher, if there are some identifiers that relate to internal data. 
- Owner and user ratings: Data is rated according to its completeness, correctness and freshness.

These criteria can be used to determine the credibility of the data sources, which affects the selection of data. With regard to internal and external data, a challenge is to identify a satisfactory tradeoff between the quality and quantity of the retrieved data [20]. In a SSBI system, not all the data has to be stored in the data warehouse. For example, external operational data, such as weather, geographic data, etc., may be important for decision-making purposes, but does not necessarily have to be stored in a data warehouse [2].

A key ingredient for SSBI implementation is to ensure the quality of the data from different sources [20]. A challenge to overcome is associated with enabling users to select data based on quality criteria, such as accuracy, freshness, completeness, reliability, etc. [20].

Meyers [16] provides an example where the quality of the data led to problems with the analyzed result. Without warning, a previously used database table was replaced and the old table was no longer updated, which led to incorrect results in the presented report [16].

\section{Challenge \#1.3: Use correct data queries}

When the data has been cleaned, stored and made available, the challenge of making the data accessible has to be addressed, in order to implement SSBI. In a traditional BI system, a power user selects the data required to fulfil the requests from casual users. To correctly analyze the data, power users formulate queries manually to ensure the correct join of the data, which is a complex process. It is very important that queries are correct. Even the simplest mistake, e.g., no proper join between data, can compromise the analysis of the data, which can lead to decisions based on faulty information. When formulating queries, a beneficial way of properly using data from different sources is to apply metadata (also referred to as a semantic layer). The use of metadata to properly formulate queries from different sources ensures that errors are avoided [15, 20].

\section{Challenge \#1.4: Control of data integrity, security and distribution}

According to Logi Analytics [1], spreadsheets are the primarily BI tool used by organizations. However, this is one example that can cause management problems. Since the use of spreadsheets can entail a lack of control regarding the integrity, security and distribution of the data, this issue must be addressed, in order to implement SSBI [1,3]. The importance of ensuring integrity and security increases exponentially, as more external data becomes available. The aim is to make sure the data is incorporated into the data warehouse properly, without any errors. A challenge to overcome in implementing SSBI is deciding who can use and add data, how long data should be stored, and determining the minimal data quality requirements. Otherwise, SSBI may suffer from inconsistent data security and quality that can lead to problems with the access and analysis of data $[3,6]$

\section{Challenge \#1.5: Define policies for data management and data governance}

Organizations that implement SSBI, thus enabling users to access and analyze data as desired, need to establish policies for data governance that define the data available for access, how it can be accessed and whether the standard of quality is appropriate for the desired analysis [6]. If data use and decision-making are carried out in organizations without a well-defined policy for data governance, users may not understand the value of the available data and establish their own "shadow IT system", which may lead to problems regarding access to and use of the right data [6]. When the analysis of data does not make sense, it is often the result of using the wrong data $[6,16]$. According to Meyers [16], data management and governance must be clarified differently. Data management includes policies, procedures, practices and tools that are used to improve the usage of available data. Data governance is the enforcement of data management. If SSBI is implemented without policies for data management and data governance, users may waste their time working with inconsistent reports that are based on wrong data $[7,16]$. The aim is to achieve "one version of the truth" when using BI [7]. If users have access to all the data and interpret it differently, they may use different approaches that produce different results. Policies for data management and data governance avoid these problems $[6,7,16]$.

\section{Challenge \#1.6: Prepare data for visual analytics}

Visual analytics, which enables users to select and use data as desired, is a powerful tool that can be applied to improve productivity. Such technologies help users discover analytics in their own way, which enables them to process analytics in a self-service fashion, instead of relying on power users [6]. These approaches focus on facilitating the capabilities of casual users who analyze data frequently and need better tools, in order to discover and share insights 
gained from the data on their own. Such tools offer the following: graphical visualization that emphasizes the used data, search strings for data discovery, as well as analysis in a drag and drop operation. Instead of using traditional programming expertise to select, use and visualize data, a challenge that needs to be overcome when implementing SSBI is to use technology that not only presents data in a graphical form but is also easy to operate. The aim is to let casual users select, use and visualize data correctly, without help from power users. For casual users to have the ability to create their own story, regarding how they can use data to reach a conclusion, technology that facilitates their abilities to prepare data for visualization is required $[6,8]$.

\subsection{Self-reliant users}

The following section describes the challenges identified within the main category: Self-reliant users

\section{Challenge \#2.1: Make BI tools easy to use}

A challenge to overcome is to ensure that BI tools can be easily used by non-technical users [2, 3, 15]. According to Imhoff and White [2], 78\% of their 587 respondents rated this challenge as very important; which was also considered one of the highest ratings of the four objectives of SSBI. If BI tools are not easy to use and do not support user requirements, users tend to develop their own limited solutions, which are not always correct [15]. The aim is to abandon uniform guessing, when making decisions, by enabling casual users to employ true data-driven BI tools that are easy to use $[3,6,15]$. When casual users have been given the flexibility to choose, use and manipulate the data they need, as well as the support required to understand the underlying algorithms, they are able to make better decisions on time, which improves business productivity. Users can thus more freely conduct analyses in more depth, without help from power users $[2,15]$.

\section{Challenge \#2.2: Make BI results easy to consume and enhance}

For users to be self-reliant and creative, when using BI tools, they must be able to understand the information presented to them [2, 3]. Therefore, BI tools must be easy to use and must present results in the format, device and interface desired by the user. BI tools should be provided for non-technical casual users that prefer working with visuals rather than source code $[2,6]$. Casual users do not want to spend unnecessary time analyzing reports to interpret their meaning [2]. Instead, they need to understand what kind of data is available and what they can access. In addition, semantic terms and definitions that explain what kind of data is required for a specific analysis are also needed. Moreover, it should be flexible and easy to manipulate data as desired [2, 15]. Using SSBI does not imply that a user can freely incorporate data, without any rules, into a spreadsheet or ghost IT system. Even though casual users can access all data, they must be aware of the kind of data that should be used for the decision at stake. Otherwise, correct solutions are not achieved, which may lead to decisions based mostly on guesses. Experiences show that basing decision-making on guesses often leads to incorrect results[15].

\section{Challenge \#2.3: Give the right tools to the right user}

Different users have different skills and require different tools depending on their tasks, for example: accessing pre-defined reports, or data, or creating new reports [3,5]. Giving the same BI tools to both power and casual users leads to failure. Casual users either find the tools too complex to use, or power users find them too limited and inflexible [5]. As more flexibility is offered to users, the more BI skills they will require. Therefore, SSBI does not require that all its users have the same tools and access to the same functions [3]. According to Eckerson [5] and the survey which had 234 respondents, it is a big mistake to buy a single SSBI tool and let all users have access to it. There is no “one size fits all” strategy. Consequently, a major challenge is finding the balance between flexibility and complexity which enables users to be self-reliant and not doomed to wait for help [3, 5, 6]. All users should be able to apply their creativity and access the data they require without technical restrictions. Therefore, a good strategy is to offer the right tools to the right users, based on their requirements. The challenge is to understand the capabilities of the BI tools and the user requirements $[3,5,6,21]$.

\section{Challenge \#2.4: Educate users on how to select, interpret and analyze data to make decisions}

One of the biggest challenges of SSBI, according to Stodder [6], is that it requires more education and training than expected. Only $64 \%$ of the surveyed respondents gave the SSBI initiative a grade of average or worse. Novice users of BI gave a lower grade, compared to more advanced BI users. Casual users found the tools too difficult to use, while power users needed more support in order to become skilled BI developers [6]. Another problem is that users are eager to find arguments that support their results, instead of looking for errors in their own development [8]. 
Consequently, a major challenge is to provide the education and training required to become proficient at analytics. The aim is to educate and encourage users to take advantage of more advanced analytics in their work and tools, by teaching them how to validate their analyses and when to adapt data in order to compare results $[6,8]$. The idea is not to teach users how to use BI tools, but to focus instead on choosing and interpreting data on the basis of the type of analysis needed. Users should strive to become better at conducting more advanced analytics $[5,6,8]$.

\section{Discussion}

In this literature review, we explore the challenges associated with implementing SSBI, which have been identified and discussed in the current literature. Based on our findings, two main categories of challenges are presented. In this section, we discuss the implications of our findings for SSBI implementation practitioners and elaborate on avenues for future research.

The main difference between SSBI and traditional $\mathrm{BI}$ is that SSBI simplifies the process of traditional BI use. It thereby enables casual users to be self-reliant and less dependent on power users, when accessing the data used for advanced analytics and decision-making. Traditional BI is not a single software or technology that can solve all problems easily. Instead, according to the definition in [22, page 2]: "BI combines products, technology, and methods to organize key information that management needs to improve profit and performance. More broadly, we think of BI as business information and business analyses within the context of key business processes that lead to decisions and actions and that result in improved business performance."

SSBI is presented in the literature as the future and a lasting trend that promises more benefits than traditional BI. Some worth mentioning follow [1, 2, 3]:

- Users conduct their own analysis: Empowered casual users can access data more easily and with more self-reliance for the purpose of making decisions. Instead of asking power users for alternative reports, users can analyze data on their own.

- Shift from reactive to proactive: Stored data becomes old. Since SSBI facilitates the access of data, users can gain insight as soon as data arrives. Proactive decision-making can help organizations make decisions on time, which can increase market shares, cut costs, and aid expansions in many different ways, etc.

- Relieve the pressure on the IT department: More and more data is becoming available for decision- making. In a traditional BI system, power users support casual users by creating reports that are used for analysis and decision-making. If casual users want to analyze such reports from a different angle, they ask the power users to amend the report as desired. Since many users apply BI, a lot of pressure is put on the IT department and its power users. SSBI empowers casual users and enables them to perform this process on their own, thereby relieving the pressure on power users.

- Eliminate guessing when making decisions: Making decisions on time requires a good foundation, such as a report or dashboard that visualizes the underlying data required for the decision. If casual users do not understand such a report and find analyzing the data difficult, they normally have two options to choose from: request a new report from the power users or base the decision on guessing. SSBI eliminates guessing, since data can be included and amended as desired, without help from a power user.

- Save resources: Empowered casual users can perform their daily work more effectively, compared to working with traditional BI systems, which saves organizational resources.

Even though SSBI promises more benefits than traditional $\mathrm{BI}$, the existing literature on the field of SSBI is limited. Detailed discussions on how to implement SSBI are scarce. However, according to best practice reports, the interest in implementing SSBI is increasing $[1,2]$. Nonetheless, the progress of SSBI implementation is slow and problematic $[1,3,4,5,6$, 7]. For example, it is difficult to motivate organizations to implement SSBI and make the related cost investments when they have already made significant investments in traditional BI and have not achieved the desired results [7]. To gain the motivation needed to make the change to SSBI, organizations must overcome the problems related to traditional BI. SSBI will improve issues experienced with traditional BI use. The first step is to identify the challenges associated with implementing SSBI. This will motivate organizations and better prepare them for the change to SSBI.

One common mistake most organizations make is purchasing a single SSBI tool and allowing everyone access to it [5]. As previously mentioned, the most important challenge to overcome is preparing how the data will be accessed and who will use it. A recipe for disaster would be allowing all users access to a single SSBI tool and having no control of how the data is used when making decisions. Even the smallest mistake, when identifying the data and its selection criteria, can cause major problems for decision makers. 
To achieve a solid foundation for SSBI data use, welldefined policies for data management and governance are a must.

An additional significant challenge to overcome is preparing users to become self-reliant. Software with a comprehensible user interface is not necessarily a BItool that supports self-service. It is important to understand what experience and skills users require in order to use a SSBI tool. The worst case scenario is when organizations have implemented SSBI under the assumption that all the users already understand how to write SQL-queries. If users already have difficulty understanding what data sources are available, writing correct SQL-queries must be even more difficult. To overcome the challenges related to self-reliant users, organizations must understand what kind of experiences and skills users require in order to use the desired SSBI tools. Implementing SSBI is not simply a matter of installing software acquired from a vendor. Instead, working with SSBI is a process that requires more reflection from users, in order to ensure that it functions smoothly and effectively. Therefore, the first step is understanding the challenges of implementing SSBI.

\section{Future research}

There are several opportunities for further research. First, practitioners in the field of SSBI could validate the identified challenges. One option is to conduct a qualitative case study that seeks to provide in-depth knowledge regarding how organizations interpret the identified challenges. For such a case study, it would be interesting to choose a consulting firm that advises organizations that want to implement SSBI. The experiences gained from such a firm's many customer projects, involving newcomers to well-experienced BI organizations, could validate and extend the identified challenges. Another opportunity for further research would be to develop and formulate recommendations that could address each of the challenges identified.

\section{Conclusion}

The aim of this paper is to identify SSBI implementation challenges. Based on data from a literature review, ten challenges divided into two main categories have been identified.
The SSBI implementation challenges related to "Access and use of data" are:

- \#1.1: Make data sources easy to access and use.

- $\quad$ \#1.2: Identify data selection criteria.

- \#1.3: Use correct data queries.

- \#1.4: Control data integrity, security and distribution.

- \#1.5: Define policies for data management and data governance.

- \#1.6: Prepare data for visual analytics.

The SSBI implementation challenges related to "Self-reliant users" are:

- \#2.1: Make BI tools easy to use.

- \#2.2: Make BI results easy to consume and enhance.

- \#2.3: Give the right tools to the right user.

- \#2.4: Educate users in how to select, interpret and analyze data, in order to make decisions.

Based on our findings, we conclude that SSBI is not just " $a$ software to install". Instead, organizations should plan how to ensure the easy access and consumption of data, so that correct decisions can be made on time. Challenges to overcome include making the retrieval and use of data flexible and eliminating the difficulty of using multiple data sources. SSBI provides users with multiple options of data use and consumption, depending on their tasks. For users to be self-reliant, BI tools must be easy to use. Organizations must ensure that the right user has access to the particular SSBI tool required and why. Furthermore, the results obtained from the use of SSBI-tools must be easy to understand by all users. Therefore, a challenge to overcome is to understand which tools a particular user requires. Finally, the aim of SSBI is to create an environment which facilitates the access, use and sharing of data, information, reports and analyses, and enables casual users to be self-reliant, without the support of power users. The challenges presented in this paper should enable organizations to be better prepared and to avoid unnecessary obstacles in the transition from BI to SSBI.

\section{References}

[1] Logi Analytics, "State of Self Service BI Report", 2015.

[2] C. Imhoff and C. White, "Self-service Business Intelligence", Empowering Users to Generate Insights, TDWI Best practices report, TWDI, Renton, WA, 2011. 
[3] P. Alpar and M. Schulz, "Self-Service Business Intelligence", Business \& Information Systems Engineering, 2016, pp. 151-155.

[4] D. Kabakchieva, K. Stefanova and S. Yordanova, "Latest Trends in Business Intelligence System Development", Proceedings of International Conference on Application of Information and Communication Technology and Statistics in Economy and Education (ICAICTSEE), 2013, pp. 212.

[5] W. Eckerson, "Business-driven BI: Using New Technologies to Foster Self-Service Access to Insights", Tableau Software, 2012.

[6] D. Stodder, "Visual analytics for making smarter decisions faster-applying self-service business intelligence technologies to data-driven objectives", TDWI Best Practices Report, 2015.

[7] M. Weber, "Keys to sustainable self-service business intelligence", Business Intelligence Journal, 2013, pp. 18.

[8] T. V. Johannessen and A. M. Fuglseth, "Challenges of Self-Service Business Intelligence", Bibsys Open Journal Systems, 2016.

[9] C. Surajit, D. Umeshwar and N. Vivek, "An overview of business intelligence technology", Commun. ACM, 2011, pp. 88-98.

[10] B. J. Oates, Research Information Systems and Computing, Sage Publications, London, 2006.

[11] F. Rowe, "What literature review is not: diversity, boundaries and recommendations", European Journal of Information Systems, 2014, pp. 241-255.

[12] J. Webster and R. T. Watson, "Analyzing the past to prepare for the future: Writing a literature review", MIS quarterly, 2002, pp. xiii-xxiii.

[13] I. Benbasat and R. Weber, "Research Commentary: Rethinking "Diversity" in Information Systems Research", Information Systems Research, 1996, pp. 389 - 399.
[14] J. F. Wolfswinkel, E. Furtmueller and C. P. M. Wilderom, "Using grounded theory as a method for rigorously reviewing literature", European Journal of Information Systems, 2013, pp. 45-55.

[15] P. A. Schlesinger and N. Rahman, "Self-Service Business Intelligence Resulting in Disruptive Technology", Journal of Computer Information Systems, 2016, pp. 11-21.

[16] C. Meyers, "How data management and governance can enable successful self-service BI", BI Training Solutions: As Close as Your, 2014, pp. 23.

[17] M. M. Zaghloul, A. Ali-Eldin and M. Salem, "Towards a Self-service Data Analytics Framework", International Journal of Computer Applications, 2013.

[18] S. Shakti, "Business Intelligence As A Competitive Advantage", International Journal in Multidisciplinary and Academic, 2013.

[19] M. Spahn, J. Kleb, S. Grimm and S. Scheidl, "Supporting business intelligence by providing ontologybased end-user information self-service", Proceedings of the First international Workshop on ontology-Supported Business intelligence, ACM, 2008, pp. 10.

[20] A. Abelló, M. Darmont, L. Etcheverry, M. Golfarelli, J.N. Maz, F. Naumann, T. Pedersen, S.-B. Rizzi, J. Trujillo, P. Vassiliadis and G. Vossen, "Fusion Cubes: Towards SelfService Business Intelligence", Int. J. Data Warehous. Min., 2013, pp. 66-88.

[21] S. Sulaiman, J. M. Gómez and J. Kurzhöfer, "Business Intelligence Systems Optimization to Enable Better SelfService Business Users", Wsbi, 2013, pp. 35-46.

[22] S. Williams and N. Williams, "The profit impact of business intelligence", Morgan Kaufmann, 2010. 